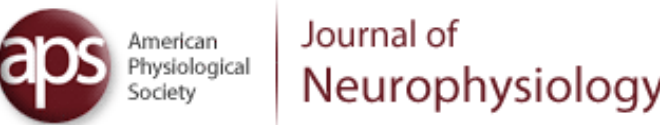

PUBLISHED ARTICLE
ARCHIVES
SUBSCRIPTIONS
SUBMISSIONS
CONTACT US

J Neurophysiol. 2015 Feb 1; 113(3): 730-739.

PMCID: PMC4312861

Published online 2014 Nov 12. doi: 10.1152/in.00645.2014

\title{
Microstimulation of area V4 has little effect on spatial attention and on perception of phosphenes evoked in area V1
}

\author{
Bruno Dagnino, ${ }^{1,{ }^{*}}$ Marie-Alice Gariel-Mathis, ${ }^{1,{ }^{*}}$ and Pieter R. Roelfsema ${ }^{\bigotimes 1,2,3}$
}

${ }^{1}$ Department of Vision and Cognition, Netherlands Institute for Neuroscience (an institute of the Royal Academy of Arts and Sciences of the Netherlands), Amsterdam, The Netherlands;

${ }^{2}$ Department of Integrative Neurophysiology, Center for Neurogenomics and Cognitive Research, VU University, Amsterdam, The Netherlands; and

${ }^{3}$ Psychiatry Department, Academic Medical Center, Amsterdam, The Netherlands

$\triangle_{\text {Corresponding author }}$

*B. Dagnino and M.-A. Gariel-Mathis contributed equally to this work.

Address for reprint requests and other correspondence: P. R. Roelfsema, Dept. of Vision and Cognition, Netherlands Inst. for Neuroscience, Meibergdreef 47, 1105 BA, Amsterdam, The Netherlands (e-mail: p.roelfsema@nin.knaw.nl).

Received 2014 Sep 2; Accepted 2014 Nov 7.

Copyright $\odot 2015$ the American Physiological Society

\section{Abstract}

Previous transcranial magnetic stimulation (TMS) studies suggested that feedback from higher to lower areas of the visual cortex is important for the access of visual information to awareness. However, the influence of cortico-cortical feedback on awareness and the nature of the feedback effects are not yet completely understood. In the present study, we used electrical microstimulation in the visual cortex of monkeys to test the hypothesis that cortico-cortical feedback plays a role in visual awareness. We investigated the interactions between the primary visual cortex (V1) and area V4 by applying microstimulation in both cortical areas at various delays. We report that the monkeys detected the phosphenes produced by V1 microstimulation but subthreshold V4 microstimulation did not influence V1 phosphene detection thresholds. A second experiment examined the influence of V4 microstimulation on the monkeys' ability to detect the dimming of one of three peripheral visual stimuli. Again, microstimulation of a group of V4 neurons failed to modulate the monkeys' perception of a stimulus in their receptive field. We conclude that conditions exist where microstimulation of area V4 has only a limited influence on visual perception.

Keywords: phosphenes, visual cortex, area V1, area V4, visual awareness, cortico-cortical feedback

THE QUESTION of how visual awareness emerges in our brain is one of the major challenges that remain to be addressed in neuroscience. There is not yet a consolidated theory of how visual percepts reach awareness (Dehaene et al. 2006; Lamme 2003; Zeki 2003). One influential theory posits that information reaches consciousness when it ignites reciprocal interactions between multiple brain areas (Edelman 1987) that allow sharing of information in a global workspace (Dehaene et al. 2003; Dehaene and Changeux 2011). Feedback connections might be important for this process, as they can amplify neuronal activity in the lower areas gating the access to consciousness (Dehaene and Changeux 2011; Lamme and Roelfsema 2000; Super et al. 2001). Neurophysiological experiments have implicated cortico-cortical feedback in visual awareness (Super et al. 2001), figure-ground segregation (Lamme et al. 1998; Super et al. 2001), and shifts of visual attention (Moore et al. 2003; Moore and Fallah 2001, 2004; Noudoost and Moore 2011; Roelfsema et al. 1998). Specifically, neuronal responses in primary visual cortex (V1) are enhanced by feedback connections if a monkey detects a figure on a background (Lamme et al. 1998; Poort et al. 2012), but this amplification does not occur if the monkey fails to perceive the figure (Super et al. 2001).

Transcranial magnetic stimulation (TMS) experiments provided additional support for the role of feedback connections in awareness. Silvanto et al. (2005) applied TMS to V1 to evoke phosphenes, artificial percepts 
of light. They showed that an additional subthreshold TMS pulse in motion-sensitive area V5 increases the probability of detecting a moving phosphene instead of a stationary one, but only if it precedes V1-TMS by $10-50 \mathrm{~ms}$, in accordance with a role of feedback connections. At the same time, V1-TMS can also interfere with the perception of phosphenes that are evoked by V5-TMS (Pascual-Leone and Walsh 2001). This interference effect was only observed if V5-TMS preceded V1-TMS, again suggesting a role for feedback connections.

Our main experiment was modeled after these TMS experiments, but we applied microstimulation (MS) in two areas of the visual cortex of monkeys. The advantage of MS is that the technique activates a more circumscribed population of neurons (Histed et al. 2009; Schiller and Tehovnik 2008; Tehovnik 1996; Tehovnik et al. 2005). Will subthreshold MS in a higher visual area influence the perception of phosphenes evoked with MS in V1? If it does, we could test the effect of V4-MS on neuronal activity in V1 to investigate how feedback connections contribute to visual awareness.

In a first experiment we applied subthreshold MS in area V4 in a task in which monkeys had to detect a phosphene evoked by MS of V1 neurons with overlapping receptive fields (RFs). We expected that V4-MS would modulate the perception of phosphenes elicited by V1-MS. To our surprise, even though feedback connections from V4 to V1 have a strength that is comparable to that of those from MT to V1 (Markov et al. 2011), we observed no effect of V4-MS on the phosphene detection threshold in V1. The activity of neurons in area V4 also provides a sensitive measure for where monkeys direct their attention (Cohen and Maunsell 2010, 2011). We therefore tested whether V4-MS influences the distribution of spatial attention across a visual stimulus in a contrast change detection task as has been observed with MS of the frontal eye fields (FEF) (Moore and Fallah 2001). However, we also did not observe any effect of V4-MS in this task. Our results imply that there are conditions where V4-MS influences neither the detection of V1-evoked phosphenes nor the distribution of visual attention.

\section{METHODS}

Surgeries and mapping. Two male monkeys (monkeys $B$ and $C$ ) participated in this study. In a first operation, a head holder was implanted. In a separate surgery, arrays of $4 \times 5$ or $5 \times 5$ electrodes (Blackrock) with a thickness of $80 \mu \mathrm{m}$ and a length of 1 or $1.5 \mathrm{~mm}$ were chronically implanted in areas $\mathrm{V} 1$ and V4. The surgical procedures were performed under aseptic conditions and general anesthesia, complied with the National Institutes of Health Guide for the Care and Use of Laboratory Animals, and were approved by the Institutional Animal Care and Use Committee of the Royal Netherlands Academy of Arts and Sciences. Details of the surgical procedures and the postoperative care have been described elsewhere (Poort et al. 2012; Super and Roelfsema 2005).

We measured the RF dimensions of every V1 recording site by determining the onset and offset of the response to a slowly moving light bar for each of four movement directions (Kato et al. 1978). V4-RFs were mapped by presenting white squares $\left(1^{\circ} \times 1^{\circ}\right)$ on a gray background at different positions of a grid $\left(1^{\circ}\right.$ spacing).

Microstimulation. For MS, we used trains of negative-first biphasic pulses of $400-\mu$ s duration (200 $\mu$ s per phase) at a frequency of $200 \mathrm{~Hz}$ through one of the electrodes in V1/V4, using a custom-made two-channel constant-current stimulator. We varied the amplitude and number of pulses (we used different numbers of pulses in the 2 tasks, see below), but all currents stayed within the range of 5-250 $\mu \mathrm{A}$. We tested the impedance of the stimulated electrodes before every session and obtained values between 40 and $280 \mathrm{k} \Omega$ (mean $107 \mathrm{k} \Omega$, SD $62 \mathrm{k} \Omega$ ). Even though unlikely, to rule out any changes in impedance over days due to the MS, we checked in monkey $C$ whether there was a significant change in impedance across sessions. The mean difference between successive sessions was $-13.7 \pm 12.6 \mathrm{k} \Omega$ (mean $\pm \mathrm{SE}$, i.e., a small decrease in impedance), but it was not significantly different from zero $\left(P>0.1, t_{11}=-1.09\right)$.

Behavioral setup. The monkeys performed both tasks while seated at a distance of $75 \mathrm{~cm}$ from a 21 -in. CRT monitor with a refresh rate of $70 \mathrm{~Hz}$ (phosphene detection task) or $100 \mathrm{~Hz}$ (dimming detection task) and a resolution of $1,024 \times 768$ pixels. The eye position was monitored with a video-based eye tracker (Thomas Recording) and sampled at $250 \mathrm{~Hz}$. A trial was initiated when the monkey had maintained his gaze for 300 ms within a (virtual) fixation window, $1.5^{\circ}$ in diameter, centered on the fixation point. The monkey obtained a juice reward at the end of each correct trial. 
Phosphene detection task. To estimate the phosphene detection threshold we used a yes/no forced-choice, delayed-saccade task (Fig. 1A). On each trial we presented a fixation point in the center of the screen (size of $0.3^{\circ}$ of visual angle) and a "catch dot" in the periphery (size of $0.3^{\circ}$ of visual angle). We randomly selected $50 \%$ of the trials as MS trials, and on those trials we delivered a train of MS pulses after $300 \mathrm{~ms}$ of fixation to elicit a phosphene at the location of the RF of the stimulated electrode. The other $50 \%$ of trials were catch trials without MS. In both conditions the monkeys were required to maintain fixation during an additional delay of $500 \mathrm{~ms}$. After this delay the fixation dot turned blue, cuing the monkeys to make a saccade. The additional delay excludes reflexive saccades that might be elicited by the direct activation of motor structures like the superior colliculus (Tehovnik et al. 2003, 2005). The monkeys reported the detection of a phosphene by making a saccade to the location of the RF and the absence of a phosphene by making a saccade to the catch dot. The trial was considered correct only if the monkey reported a phosphene in MS trials or made an eye movement to the catch dot in catch trials, and incorrect otherwise. The monkeys obtained a juice reward after every correct trial.

Before they could perform this task, the monkeys were trained on a very similar task, in which they had to report the presence of a visual stimulus that was flashed for $20 \mathrm{~ms}$ on $50 \%$ of the trials. The stimuli were presented at various positions on the screen, and we decreased their luminance as the monkeys became better in the task. Training with visual stimuli was continued until the monkeys' accuracy was higher than $80 \%$ with very dim targets. This training phase took $\sim 1.5 \mathrm{mo}$. We then replaced the visual stimuli by MS. We interleaved blocks of visual stimulation trials with blocks of V1-MS trials so that the monkeys would learn to also make saccadic eye movements to the phosphene locations. We gradually reduced the number of visual stimulation blocks until the monkeys reliably detected the V1 phosphenes. This transition from visual to electrical stimulation was completed in $2 \mathrm{wk}$. Once the V1-MS training was completed we repeated the procedure with V4-MS. First, we presented visual stimuli with different shapes (circles, ellipses, and squares), colors (red, blue, green, and gray), and sizes (between $3^{\circ}$ and $6^{\circ}$ of visual angle) so that the monkeys learned to make saccades to a variety of visual stimuli. This training phase was completed in $\sim 3 \mathrm{wk}$. In the last phase, which took $\sim 2 \mathrm{wk}$, we interleaved blocks of visual stimulation with blocks of V4-MS until the monkeys reliably detected the V4 phosphenes.

To determine the threshold for the electrical stimulation we varied the amplitude of the electrical MS, using a Bayesian adaptive psychometric method called QUEST (Watson and Pelli 1983) set to determine a threshold of $80 \%$ correct. To increase the robustness of the estimation we did not use every single correct or incorrect answer as input to the QUEST, but we introduced a counter for both correct and incorrect MS trials. Every time there was a correct or incorrect MS trial, we incremented the corresponding counter. Once either of the counters reached a value of 2, the QUEST was updated and both counters were set to 0 . The update of the QUEST was done in the following manner: if the correct trial counter reached 2 first, then the QUEST was fed with a value of 1, meaning that it should decrease the amplitude of MS. If the counter of incorrect trials was the first one to reach a value of 2, the QUEST was fed with a value of 0 , indicating that it should increase the current. After 15 reversals (a reversal being a change in the monotonicity of the series of successive current amplitudes) the QUEST stopped and the threshold was computed as the mean of the last 10 reversals.

In each session we tested a combination of one V4 electrode and one V1 electrode with overlapping RFs. First we measured the threshold for phosphene detection for the V4 electrode, with three (monkey $C$ ) or four (monkey $B)$ pulses with a separation of $5 \mathrm{~ms}(200 \mathrm{~Hz})$. We used one pulse extra in monkey $B$ to keep the current thresholds below $100 \mu \mathrm{A}$. We here used a small number of pulses in V1 and V4 to keep the stimulus temporally concise, which is beneficial for comparing the results to the previous TMS studies, which used a single pulse per brain region.

The average V4 threshold was $51 \pm 33 \mu \mathrm{A}$ (mean $\pm \mathrm{SD}$ ). We then determined the phosphene detection threshold for the electrode in V1 (5 pulses). Of all trials, 50\% were V1-MS trials and the other 50\% were catch trials without V1-MS. There were 11 conditions for V1-MS trials. Ten of these conditions combined the train of pulses in V1 (evoking the phosphene) with V4-MS at 50\% of the V4 threshold (measured at the start of the session) at different stimulus-onset asynchronies (SOAs) so that V4-MS either preceded or followed V1-MS. V4-MS was between -68 ms and $22 \mathrm{~ms}$ after V1-MS in steps of $10 \mathrm{~ms}$ ("V4-V1" conditions). The 11th condition monitored the phosphene detection threshold in V1 without V4 stimulation 
("V1-only" condition). The average threshold in this condition was $32 \pm 18 \mu \mathrm{A}$. These 11 QUEST procedures ran in parallel, in a randomly interleaved fashion.

Of the catch trials, $90 \%$ were without any MS and the animal was required to make a saccade to the catch dot. In the other $10 \%$ of catch trials we only stimulated V4 to investigate whether the V4-MS at $50 \%$ of the threshold could elicit a phosphene by itself. In these trials the monkeys were also rewarded for eye movements to the catch dot, just as in the regular catch trials, and no reward was given for making a saccade toward the RF of the V4 stimulation site. We refer to subthreshold V4-MS trials in which the monkey made an erroneous saccade to the overlapping V1/V4-RFs as "V4 false alarm responses." The monkeys were required to only make saccades to the V1/V4-RF location upon V1-MS. To investigate the influence of V4-MS on catch trials, we computed the standard false positive rate (FPR) on regular catch trials as the percentage of catch trials in which the monkeys reported a phosphene and the V4-FPR as the percentage of subthreshold V4-MS trials in which monkeys reported a phosphene.

We collected a total of 15 sessions in monkey $B$ and 16 sessions for monkey $C$. On average, every session had $815 \pm 108$ trials. Figure $2 A$ illustrates the V1-RFs that have been superimposed on the V4-RFs. On every test day we used a different electrode combination. For each SOA, threshold values either lower or higher than 5 times the standard error across sessions were considered outliers and removed from the analysis. On average, $15 \%$ of the single-session thresholds were removed.

d-Prime. To compute the $d$-prime we used the MATLAB toolbox Palamedes (Prins and Kingdom 2009). First, for each session, we fitted a psychometric function for both V1-only (the data from the threshold with stimulation in V1 only) and V4-V1 (the data from all the V4-V1 SOAs together) conditions with a logistic function, adding the FPR (for the V1-only condition) and V4-FPR (for the V4-V1 condition) as data points with zero current, providing an accurate measure of the false alarm rate. Thus the psychometric functions expressed $d$-prime as a function of stimulation current for every session, and we then averaged across sessions, clipping $d$-prime values larger than 4 to a value of 4 because these very high $d$-prime values depended strongly on the presence of one or a few errors.

Dimming detection task. To test the influence of V4-MS in a distributed attention task, we trained the monkeys to perform a three-alternative forced-choice task, in which they had to detect the dimming (transient decrease in luminance) of one of three bars $\left(5^{\circ} \times 0.21^{\circ}\right.$ of visual angle). One bar was presented in the RF of one of the V4 electrodes and the other two at the same eccentricity but at a position rotated by $120^{\circ}$ around the fixation point. We also presented line elements in the background. These background line elements had the same luminance of $47.6 \mathrm{~cd} / \mathrm{m}^{2}$ as the target bars (background: $11 \mathrm{~cd} / \mathrm{m}^{2}$ ) and a length of $1^{\circ}$, a width of $0.05^{\circ}$, and randomized orientation and were spaced by $0.5^{\circ}$ in both vertical and horizontal directions, with a randomized jitter on their central position based on a normal distribution with a SD of $0.2^{\circ}$. The background line elements covered the whole screen except from a region of $1^{\circ}$ surrounding the three target bars. We started each session by measuring the V4 phosphene threshold with 20 pulses (200 $\mathrm{Hz}$, total duration $100 \mathrm{~ms}$ ) at the tested V4 electrode. After the initial $300 \mathrm{~ms}$ of fixation, we presented the three bars and the background (Fig. $3 A$ ). The task of the monkey was to detect the dimming of one of the three bars by making an eye movement to it between 100 and $500 \mathrm{~ms}$ after the dimming occurred. Reaction times shorter than $100 \mathrm{~ms}$ were counted as false alarms. All bars were equally likely to dim, but only one did so on each trial, after a random time between 200 and $900 \mathrm{~ms}$ after the presentation of the stimulus. The dimming lasted for $100 \mathrm{~ms}$, and on MS trials ( $50 \%$ of the trials) the dimming took place immediately after the 100-ms MS train applied to a V4 electrode with a RF that overlapped with one of the bars (comparable to Moore and Fallah 2001). We used an amplitude that was half of the V4 phosphene detection threshold. We also carried out a few sessions with suprathreshold V4 stimulation. In these sessions we set the current at $200 \%$ of the V4 phosphene detection threshold.

Because the temporal expectation of an event can modulate perceptual processing (Vangkilde et al. 2012), we maintained a constant hazard rate for the dimming event. Specifically, we used an exponential distribution of dimming times between 200 and $900 \mathrm{~ms}$. In addition, 10\% of the trials were "long" trials with a dimming time of 1,200 ms. As a result, the hazard rate was constant between 200 and $900 \mathrm{~ms}$. If the dimming had not yet occurred at $900 \mathrm{~ms}$, however, it always occurred at 1,200 ms, but these long trials were not included in the analysis. 
During the experiment we measured the luminance change threshold for detection of the dimming (in \% of bar luminance) with and without MS, separately for each bar. We used a 3 up/ 1 down staircase procedure, and the 6 staircases ( 3 bars $\times$ MS or no MS) were run in parallel (with trials interleaved). The gammacorrected RGB luminance of the bar was $47.6 \mathrm{~cd} / \mathrm{m}^{2}$, and the eight luminance values for the dimmed bars were $29.4,31.9,34.3,36.7,39.2,41.6,44$, and $46.4 \mathrm{~cd} / \mathrm{m}^{2}$.

Statistical tests. All statistical tests were performed with MATLAB standard functions. Unless noted otherwise, all $t$-tests were one-sample, two-tailed tests. Paired $t$-tests were also two-tailed. We used a oneway ANOVA to assess the influence of SOA in the phosphene detection task.

\section{RESULTS}

Influence of V4 microstimulation on phosphenes induced in area V1. Our first experiment investigated how interactions between areas of the visual cortex influence the detection of phosphenes and was modeled after previous TMS studies on the interaction between higher and lower areas of the visual cortex (PascualLeone and Walsh 2001; Silvanto et al. 2005). We trained two monkeys to detect a phosphene elicited by suprathreshold MS of an electrode of one of the chronically implanted arrays in V1 and to report the phosphene by making a delayed saccade to its spatial location. We paired V1-MS with subthreshold MS of an electrode of a V4 array where neurons had overlapping RFs, at various SOAs. We tested the interaction between MS in V1 and V4 in 15 combinations of V1 and V4 electrodes in monkey $B$ and in 16 combinations in monkey $C$ (the neurons' RFs are shown in Fig. $2 A$ ).

At the start of every experimental session, we determined the threshold of V4-MS that evoked a phosphene and we then set the V4-MS intensity at $50 \%$ of that threshold for the remainder of the session. This choice of $50 \%$ of the threshold was inspired by previous studies using MS in frontal cortex showing that this current level can induce changes in behavior (Moore and Fallah 2001). The average V4 threshold was $51 \pm$ $33 \mu \mathrm{A}$ (mean $\pm \mathrm{SD}$ ). The threshold in V1 for evoking phosphenes with the electrode arrays (in the absence of V4-MS) was $32 \pm 18 \mu \mathrm{A}$, which is slightly higher than thresholds obtained with acutely inserted electrodes (Tehovnik et al. 2005).

We next investigated the influence of the subthreshold V4-MS on the phosphene threshold in V1 at various SOAs between V1-MS and V4-MS. Figure $1 B$ shows an example electrode combination from a session with monkey $C$. In this session, the V4 stimulation threshold was $38.3 \mu \mathrm{A}$ (we therefore stimulated with 19 $\mu \mathrm{A}$ ) and the threshold in V1 in the absence of V4-MS was $27.3 \mu \mathrm{A}$. Figure $1 C$ illustrates the sequence of applied currents according to the QUEST staircase for V1-only (in the absence of V4-MS) and combined V1-V4 stimulation trials with an SOA of $-38 \mathrm{~ms}$ (i.e., V4-MS preceding V1-MS by $38 \mathrm{~ms}$ ). The MS threshold in V1 for the $-38 \mathrm{~ms}$ SOA was $22.7 \mu \mathrm{A}$. Thus, in this particular example, V4-MS led to a small reduction in $\mathrm{V} 1$ threshold.

To quantify the influence of V4-MS on the phosphene threshold in V1, we computed the phosphene threshold ratio (PTR) for each SOA, defined as the ratio between the threshold for phosphene detection with combined V1/V4-MS and the threshold with V1-MS only. Thus a PTR value of 1 means that V4-MS had no effect on V1 phosphene detection thresholds, a value higher than 1 indicates that V4-MS impaired V1 phosphene detection, and a value lower than 1 indicates that V4-MS facilitated V1 phosphene detection. In the example session illustrated in Fig. 1D, PTR values were lower than 1 across all SOAs, indicating that V4-MS produced a general decrease of the MS threshold in V1.

To investigate the mean influence of V4-MS on V1 phosphene thresholds, we averaged values across SOAs to compute the $\mathrm{PTR}_{\text {Mean }}$ for every session ( $n=15$ for monkey $B$ and $n=16$ for monkey $C$; Fig. $2 B$ ). The PTR $\mathrm{P}_{\text {Mean }}$ was significantly lower than 1 for monkey $B\left(P<0.05, t_{14}=-6.34\right)$, and there was a trend in the same direction in monkey $C\left(P<0.1, t_{15}=-1.75\right)$. Thus V4-MS caused a general decrease in V1-MS thresholds. Inspection of the individual data points in Fig. $2 B$ suggests that this effect is relatively independent of the SOA, although previous studies suggested that V4-MS might interact most strongly with V1 phosphene detection if it precedes V1-MS. We therefore specifically tested the influence of SOA on the PTR with a one-way ANOVA but did not observe a significant effect of SOA on the PTR in either monkey $\left(F_{9,127}=0.41, P>0.1\right.$ in monkey $C ; F_{9,117}=1.62, P>0.1$ in monkey $\left.B\right)$. Thus the effect of V4-MS on V1 phosphene detection did not depend on SOA, although we observed a general decrease in phosphene detection thresholds (Fig. 2B). We also tested whether the interaction between MS in V1 and V4 depended 
on the distance between RFs of neurons at the stimulation sites. To this aim, we analyzed the $50 \% \mathrm{~V} 1-\mathrm{V} 4$ combinations with nearest and the $50 \%$ with farthest RFs separately but did not observe a significant effect of RF distance on the PTR (ANOVA; $F_{1,118}=0.15, P>0.5$ in monkey $C$ and $F_{1,126}=0.9, P>0.3$ in monkey $B)$.

We suspected that the general increase in phosphene sensitivity might be caused by the detection of the V4MS pulses themselves. Although we set the V4-MS pulse amplitude at $50 \%$ of the threshold (see METHODS) and we did not reward the monkeys for saccades to V4 phosphenes, it is conceivable that the monkeys made saccades to phosphenes elicited by V4-MS on a fraction of the trials. To investigate the detection of V4 phosphenes, we measured a V4-FPR by delivering V4-MS in $10 \%$ of the catch trials without V1-MS and compared it to the false alarm rate on trials without MS in either area in the other $90 \%$ of catch trials. V4-MS increased the FPR from $10 \%$ to $15 \%$ in monkey $C$ (paired $t$-test; $P<0.05, t_{15}=2.8$ ) and from $8 \%$ to $23 \%$ in monkey $B\left(P<0.01, t_{14}=4.3\right)$ (Fig. $\left.2 C\right)$, which indicates that monkeys made saccades to phosphenes elicited in V4 at $50 \%$ of the threshold in some of the trials even though they were never rewarded for these saccades.

We used signal detection theory (Green and Swets 1966) to investigate whether the induction of V4 phosphenes could account for the general decrease in the apparent V1-MS threshold caused by V4-MS. A genuine increase in the sensitivity for V1 phosphenes should increase the $d$-prime, whereas the detection of V4 phosphenes should only change the criterion, i.e., a general increase in the probability of reporting the presence of a phosphene. The signal-detection analysis revealed that the $d$-prime as function of V1-MS stimulation amplitude was hardly influenced by V4-MS in either monkey (Fig. 2D). For example, the current in V1 at which $d$-prime reached a value of 2.12 did not differ between V1-only and V4/V1 combined MS trials (paired $t$-test; $P>0.1, t_{15}=1.65$ for monkey $C$ and $P>0.1, t_{14}=0.68$ for monkey $B$ ) (arrows in Fig. 2D). In contrast, the bias was significantly different between conditions (paired $t$-test; $P<$ $0.05, t_{15}=-2.86$ for monkey $C$ and $P<0.01, t_{14}=-4.8$ for monkey $B$ ). We computed a $d$-prime threshold ratio (DTR), which is equivalent to the PTR ratio, while correcting for the influence of V4-MS on the monkeys' criterion. DTR was defined as the ratio between the currents at which the $d$-prime reached a value of 1 . In this analysis we had to bin SOAs in groups of two because the number of trials per SOA bin was insufficient for a reliable determination of $d$-primes in individual sessions. As expected, the DTR $\mathrm{Mean}_{\mathrm{M}} \mathrm{did}$ not differ significantly from $1\left(P=0.71, t_{14}=0.38\right.$ in monkey $B ; P<0.8, t_{14}=0.25$ in monkey $C$; Fig. $\left.2 E\right)$. Thus, although the V4-stimulation was intended to be subthreshold, the induction of V4 phosphenes on a fraction of trials accounted for the apparent decrease in the threshold for detecting V1 phosphenes, which was independent of the SOA (Fig. 2B). These results, taken together, indicate that V4-MS did not influence the perception of $\mathrm{V} 1$ phosphenes themselves at any SOA.

Influence of V4 microstimulation on dimming detection. Despite the well-established theoretical and experimental basis for our first experiment, we did not observe a specific influence of V4-MS on the detection of phosphenes evoked in V1. One of the reasons for this negative result could be the nature of the visual percepts evoked by MS. In the previous experiment we tested whether there was an interaction between two MS trains, one in V1 and one in V4. Might MS in V4 only influence the perception of visual stimuli without an effect on phosphenes elicited in V1? Moreover, in the first experiment the target stimulus could only appear at one location that was held constant for the entire session. As a result, the monkeys' attention was presumably directed to this location, which coincided with the stimulated RF. Thus we only tested whether V4-MS influenced awareness at a specific location but not how it influenced the distribution of spatial attention. Previous studies (Cohen and Maunsell 2010, 2011) demonstrated that the activity of neurons in V4 reflects the distribution of attention on single trials because they increase their response if the RF coincides with the attended location. Is it also possible to influence the distribution of attention with V4-MS? Such an effect of MS on the distribution of selective attention would not be without precedent. In an elegant study, Moore and Fallah (2001) demonstrated that MS in the FEF influenced the performance in monkeys on a task in which they had to detect a contrast decrement of a stimulus in a crowded visual scene. They found that FEF-MS facilitated the detection of the contrast decrement, but only if the motor field of the stimulated neurons overlapped with the visual stimulus. One possible interpretation of this result is that the FEF-MS summoned spatial attention to the FEF motor field, thereby facilitating dimming detection at the corresponding location in the visual field while interfering with dimming detection at other locations. We here asked whether the activation of V4 with MS could trigger a similar 
effect. Does V4-MS facilitate or impair the detection of a small change in a visual stimulus or is there also little influence on the distribution of attention?

To address this question we trained the monkeys to detect the dimming of one of three larger bars that were presented on a background of smaller, irrelevant line elements (Fig. $3 A$ ). In half of the trials the dimming was preceded by a train of $20 \mathrm{MS}$ pulses (subthreshold as determined with the phosphene detection task) applied to a V4 electrode with a RF that overlapped with one of the bars. We measured the luminance change threshold for detection of the dimming (in \% of bar luminance) with and without MS, separately for each bar. Across days, we gathered data from $25 \mathrm{MS}$ sessions in monkey $C$ and 24 sessions in monkey B, approximately equally distributed across three V4 electrodes per monkey (6-10 sessions per electrode; Fig. 4A).

Does V4-MS decrease the detection threshold of the dimming at the V4-RF, perhaps at the expense of higher thresholds at other retinotopic locations? Figure 3, $B-D$, illustrates the effect of V4-MS on the dimming detection threshold in an example session. One of the three target bars was placed in the RF of the stimulated V4 electrode (Fig. $3 B$ ). It can be seen that the staircases for the bar at the RF location in the presence and absence of V4-MS converged to a similar value of $\sim 8.6 \%$ for the luminance decrement ( Fig. $3 C$ ). We measured the dimming detection threshold condition as the average decrement for the last 10 reversals (Fig. 3D) but did not observe a significant difference between the MS and the blank condition in the example session (comparison of the last 10 reversals of the staircase, $P>0.1, t_{18}=0.59$ ).

We compared the contrast decrement detection thresholds with and without V4-MS across all sessions in both monkeys (i․ $4 B$ ). The average thresholds for the RF bars were $7.7 \%$ and $5.5 \%$ (monkeys $C$ and $B$, respectively) without V4-MS and 7.9\% and 5.3\% with V4-MS. For the bars outside the RF, the average thresholds were $8.9 \%$ and $6.6 \%$ with V4-MS and $9.1 \%$ and $5.4 \%$ without V4-MS. These thresholds were lower than found by Moore and Fallah (2001), presumably because they used flashing distractors whereas our distractors had a constant luminance. MS did not have a significant influence on the threshold of dimming detection for any of the bars (paired $t$-test with Bonferroni correction for testing 2 types of bars, RF bars and non-RF bars: monkey $B$ : RF bar $P>0.05, t_{23}=-2.0$; non-RF bars $P>0.1, t_{47}=0.91$; monkey $C$ : RF bar $P>0.1, t_{24}=0.57$; non-RF bars $\left.P>0.1, t_{49}=-0.94\right)$. Thus V4-MS influenced dimming detection neither in the RF of the stimulated neurons nor at the other locations remote from the RF. We next investigated the possibility that MS influenced the reaction time. In monkey $B$, the average reaction time across sessions if the RF bar dimmed was $232 \pm 16 \mathrm{~ms}$ (mean $\pm \mathrm{SE}$ ) in the MS condition and $235 \pm 11$ $\mathrm{ms}$ without MS, a difference that was not significant (paired $t$-test, $P>0.1, t_{23}=-1.3$ ). If one of the bars outside the RF dimmed, the average reaction time was $212 \pm 10 \mathrm{~ms}$ in the MS condition and $212 \pm 11 \mathrm{~ms}$ without MS (paired $t$-test, $P>0.1, \mathrm{df}=23, t_{47}=-0.02$ ). Similar results were obtained in monkey $C$, with a mean reaction time of $276 \pm 17 \mathrm{~ms}$ with MS and $276 \pm 17 \mathrm{~ms}$ without MS if the dimming bar fell in the RF (paired $t$-test, $P>0.1, t_{24}=-0.1$ ). If one of the other two bars dimmed, the mean response time was $258 \pm$ $12 \mathrm{~ms}$ with V4-MS and $257 \pm 13 \mathrm{~ms}$ without MS (paired $t$-test, $P>0.1, t_{49}=0.59$ ). Thus subthreshold MS in area $\mathrm{V} 4$ did not influence response time in the dimming detection task either.

In the V1-V4 MS experiments described above we observed a general increase in the false alarm rate with subthreshold V4-MS, which suggested that it caused phosphenes in a small fraction of the trials. We used similar current levels in the dimming detection task, and it is therefore conceivable that the monkeys also perceived phosphenes in some of the MS trials. We do not know whether V4 phosphenes decrease or improve dimming detection. On the one hand, phosphenes could act as an exogenous attention cue to improve performance at the MS location. On the other hand, they could act as a mask that interferes with dimming perception. Although the observed null effect in the first experiment argues against either possibility, we applied higher currents in a few experiments to test the effect of V4 phosphenes on dimming detection.

We ran three sessions in monkey $B$ and two in monkey $C$ with MS currents set at $200 \%$ of the phosphene detection threshold. In monkey $C$ we did not observe an effect of V4-MS on contrast detection. However, we did observe a consistent effect in monkey $B$ across the three sessions. MS in V4 increased the dimming detection threshold for the bar that was in the RF (overlap; Fig. 4C) and reduced the threshold for bars outside the RF (no overlap; Fig. 4C). This result demonstrates that stronger V4 phosphenes interfered with dimming detection in monkey $B$. 


\section{DISCUSSION}

In the present study we tested the hypothesis that neuronal activity in V4 may gate the access of neuronal activity in lower visual areas into awareness or may cause shifts of visual attention. To this aim we electrically stimulated V4 and probed the effects on the perception of phosphenes evoked in V1 or on the perception of a contrast change of a visual stimulus. We did not observe an effect of V4-MS on behavioral thresholds in either task, which implies that subthreshold MS in V4 has a strong impact neither on the sensitivity for V1-induced phosphenes nor on the distribution of attention.

Influence of V4 microstimulation on detection of phosphenes evoked in V1. MS in area V4 had little influence on the threshold for the detection of phosphenes that were elicited by MS in V1. The absence of an interaction between MS in V1 and V4 may at first sight seem unexpected if compared to previous studies that used one TMS coil to evoke phosphenes in V1 of human observers and another TMS coil to stimulate motion-sensitive area MT (Pascual-Leone and Walsh 2001; Silvanto et al. 2005). These previous studies demonstrated that a suprathreshold TMS pulse in area V1 interacts with a subthreshold TMS pulse applied to motion-sensitive area MT so that the threshold for motion perception is reduced, but only if MT stimulation precedes V1 stimulation. This effect was strongest if the delay between MT and V1 TMS was $\sim 50 \mathrm{~ms}$, which suggested the involvement of feedback connections.

Here we did not observe such a delay-specific influence of V4-MS on the threshold for phosphenes evoked by V1 stimulation but only a general decrease of the threshold that was compatible with the detection of the V4 phosphenes on a fraction of trials. There are a number of differences between the previous TMS studies and the present study that could explain the disagreement. The most notable difference is the method used to influence neuronal activity in the cortex. TMS stimulates a large region of cortex of several millimeters to centimeters (Walsh and Cowey 1998, 2000). In contrast, MS influences a much smaller region of $1 \mathrm{~mm}$ or less around the tip of the electrode (Histed et al. 2009, 2012; Tehovnik 1996) and permits a much more precise control over the retinotopic overlap of stimulated neurons in the two brain areas. In the present study we only selected pairs of recording sites with overlapping RFs, as V4 neurons with RFs that overlap with those in V1 are predicted to exert a stronger control over V1 activity than V4 neurons with RFs that do not overlap. This difference between the present MS study and previous TMS studies raises the possibility that retinotopically nonoverlapping regions targeted by TMS contributed to the interaction between the two areas. Another difference is that the TMS experiments observed that activation of MT influenced the sensory quality of the perceived phosphene rather than the threshold itself. Because we only tested whether feedback from V4 influenced the threshold of phosphenes induced in V1, we cannot rule out the possibility that altered feedback from V4 changed the sensory qualities of the phosphene, like its perceived shape or color. It is even possible that V4 stimulation only influences awareness if the shape preferred by the stimulated V4 neurons matches the shape of phosphene elicited in V1. These matches are presumably more likely with TMS because it influences larger regions of the cortex, although we increased the probability of matches by ensuring that the RFs in V1 and V4 were overlapping. A final difference between studies is that we stimulated V4, whereas the TMS studies targeted motion-sensitive area MT. We do not know whether our double MS approach would have revealed an interaction between V1 and MT, but the strength of the feedback projection from V4 to V1 is similar to the strength of the projection from MT to V1 (Markov et al. 2011), so that the differences between techniques seem to be a more likely cause of the discrepancy.

Influence of V4 microstimulation on distribution of attention. We also investigated whether V4-MS influences the distribution of attention, because previous studies demonstrated that activity in V4 reflects where a monkey directs attention on single trials (Cohen and Maunsell 2010, 2011). Our second experiment therefore paired subthreshold MS in V4 with the dimming of a visual stimulus. We did not observe an effect on the detection of a dimming stimulus, thus demonstrating that subthreshold V4-MS does not alter the perception of subtle contrast changes of visual stimuli. Previous experiments with related paradigms showed that MS of FEF does influence dimming detection, in a manner that can be explained by an attention shift (Moore et al. 2003; Moore and Fallah 2001, 2004), and that MS in area LIP biases eye movement decisions toward the RF of the stimulated neurons (Dai et al. 2014). However, despite using related paradigms as well as similar stimulation current levels (mean of $20 \mu \mathrm{A}$ in our experiment vs. median of $10 \mu \mathrm{A}$ in Moore and Fallah 2001 and $40 \mu \mathrm{A}$ in Dai et al. 2014), our second experiment also failed to reveal an effect of MS on the threshold in a dimming detection task. This second null result suggests that the influence of V4 stimulation on covert attention is limited. In contrast, stimulation of FEF 
or LIP appears to trigger feedback mechanisms to the visual cortex that influence the spatial distribution of attention and eye movement control (Dai et al. 2014; Moore et al. 2003). This influence of MS in FEF or LIP is consistent with the previously proposed role of frontal and parietal cortex in overt and covert shifts of attention (Rossi et al. 2009). In contrast, the present results demonstrate that MS of V4 does not cause a shift of attention, which suggests that it is more involved in visual processing than in the control of spatial attention.

Possible influences of feedback on perception and distribution of attention. Although we did not observe an influence of V4-MS on V1 phosphene thresholds, our results do not exclude the possibility that V4-MS changed the perceptual quality of the phosphenes. Schiller et al. (2011) recently trained monkeys to report the size, color, and brightness of phosphenes evoked by cortical MS. They provided evidence that V1 phosphenes evoked by currents in the same range used here have a small size $\left(<0.5^{\circ}\right)$, a relatively weak contrast $(<10 \%)$, and in some cases a specific color. Future studies could use the double MS paradigm to test the influence of MS in higher visual areas on the perceived quality of phosphenes evoked in area V1. For instance, it is conceivable that subthreshold stimulation of cells tuned for color in V4 could change the perceived color or shape of phosphenes evoked by MS in area V1. A similar argument can be made about the absence of an effect of V4 stimulation on the distribution of attention. We did not obtain evidence that V4-MS influences the distribution of spatial attention but cannot exclude that V4 can exert an influence on feature-based attention. For example, MS of V4 neurons tuned to red might lead to a specific improvement in the detection of the dimming of red bars relative to the dimming of green bars. A drawback of our negative results is that we cannot exclude that V4-MS would have influenced the perception of dimming of stimuli had we used different colors, shapes, or sizes. Future experiments that test the influence of MS on the perception of specific features might further our understanding of the functional role of feedback connections between extrastriate cortex and area V1.

\section{GRANTS}

The work was supported by NWO (VICI scheme and Brain and Cognition Grant No. 433-09-208) and the European Union Seventh Framework Program (Project 269921 "BrainScaleS," Marie-Curie Action PITNGA-2011-290011 “ABC,” and ERC Grant Agreement No. 339490) awarded to P. R. Roelfsema.

\section{DISCLOSURES}

No conflicts of interest, financial or otherwise, are declared by the author(s).

\section{AUTHOR CONTRIBUTIONS}

Author contributions: B.D., M.-A.G.-M., and P.R.R. conception and design of research; B.D. and M.-A.G.-M. performed experiments; B.D. analyzed data; B.D., M.-A.G.-M., and P.R.R. interpreted results of experiments; B.D. and P.R.R. prepared figures; B.D. and P.R.R. drafted manuscript; B.D. and P.R.R. edited and revised manuscript; B.D. and P.R.R. approved final version of manuscript.

\section{ACKNOWLEDGMENTS}

We thank Kor Brandsma and Anneke Ditewig for biotechnical assistance.

\section{REFERENCES}

Bullier J, McCourt ME, Henry GH Physiological studies on the feedback connection to the striate cortex from cortical areas 18 and 19 of the cat. Exp Brain Res 70: 90-98, 1988. [PubMed: 3402571]

Butovas S, Schwarz C Spatiotemporal effects of microstimulation in rat neocortex: a parametric study using multielectrode recordings. J Neurophysiol 90: 3024-3039, 2003. [PubMed: 12878710]

Cohen MR, Maunsell JH A neuronal population measure of attention predicts behavioral performance on individual trials. J Neurosci 30: 15241-15253, 2010. [PMCID: PMC3045704] [PubMed: 21068329]

Cohen MR, Maunsell JH When attention wanders: how uncontrolled fluctuations in attention affect performance. J Neurosci 31: 15802-15806, 2011. [PMCID: PMC3579494] [PubMed: 22049423]

Dai J, Brooks DI, Sheinberg DL Optogenetic and electrical microstimulation systematically bias visuospatial choice in primates. Curr Biol 24: 63-69, 2014. [PubMed: 24332543] 
Dehaene S, Changeux JP Experimental and theoretical approaches to conscious processing. Neuron 70: 200 -227, 2011. [PubMed: 21521609]

Dehaene S, Changeux JP, Naccache L, Sackur J, Sergent C Conscious, preconscious, and subliminal processing: a testable taxonomy. Trends Cogn Sci 10: 204-211, 2006. [PubMed: 16603406]

Dehaene S, Sergent C, Changeux JP A neuronal network model linking subjective reports and objective physiological data during conscious perception. Proc Natl Acad Sci USA 100: 8520-8525, 2003. [PMCID: PMC166261] [PubMed: 12829797]

Edelman GM. Neural Darwinism: The Theory of Neuronal Group Selection. New York: Basic Books, 1987.

Girard P, Hupe JM, Bullier J Feedforward and feedback connections between areas V1 and V2 of the monkey have similar rapid conduction velocities. J Neurophysiol 85: 1328-1331, 2001.

[PubMed: 11248002]

Green DM, Swets JA Signal Detection Theory and Psychophysics. New York: Wiley, 1966.

Histed MH, Bonin V, Reid RC Direct activation of sparse, distributed populations of cortical neurons by electrical microstimulation. Neuron 63: 508-522, 2009. [PMCID: PMC2874753] [PubMed: 19709632]

Histed MH, Ni AM, Maunsell JH Insights into cortical mechanisms of behavior from microstimulation experiments. Prog Neurobiol 103: 115-130, 2012. [PMCID: PMC3535686] [PubMed: 22307059]

Kaas JH, Lyon DC Pulvinar contributions to the dorsal and ventral streams of visual processing in primates. Brain Res Rev 55: 285-296, 2007. [PMCID: PMC2100380] [PubMed: 17433837]

Kato H, Bishop PO, Orban GA Hypercomplex and simple/complex cell classifications in cat striate cortex. J Neurophysiol 41: 1071-1095, 1978. [PubMed: 702188]

Lamme VA. Why visual attention and awareness are different. Trends Cogn Sci 7: 12-18, 2003. [PubMed: 12517353]

Lamme VA, Roelfsema PR The distinct modes of vision offered by feedforward and recurrent processing. Trends Neurosci 23: 571-579, 2000. [PubMed: 11074267]

Lamme VA, Super H, Spekreijse H Feedforward, horizontal, and feedback processing in the visual cortex. Curr Opin Neurobiol 8: 529-535, 1998. [PubMed: 9751656]

Markov NT, Misery P, Falchier A, Lamy C, Vezoli J, Quilodran R, Gariel MA, Giroud P, Ercsey-Ravasz M, Pilaz LJ Weight consistency specifies regularities of macaque cortical networks. Cereb Cortex 21: 1254-1272, 2011. [PMCID: PMC3097985] [PubMed: 21045004]

Moore T, Armstrong KM, Fallah M Visuomotor origins of covert spatial attention. Neuron 40: 671-683, 2003. [PubMed: 14622573]

Moore T, Fallah M Control of eye movements and spatial attention. Proc Natl Acad Sci USA 98: 1273 -1276, 2001. [PMCID: PMC14744] [PubMed: 11158629]

Moore T, Fallah M Microstimulation of the frontal eye field and its effects on covert spatial attention. J Neurophysiol 91: 152-162, 2004. [PubMed: 13679398]

Movshon JA, Newsome WT Visual response properties of striate cortical neurons projecting to area MT in macaque monkeys. J Neurosci 16: 7733-7741, 1996. [PubMed: 8922429]

Noudoost B, Moore T Control of visual cortical signals by prefrontal dopamine. Nature 474: 372-375, 2011. [PMCID: PMC3117113] [PubMed: 21572439]

Pascual-Leone A, Walsh V Fast backprojections from the motion to the primary visual area necessary for visual awareness. Science 292: 510-512, 2001. [PubMed: 11313497]

Poort J, Raudies F, Wannig A, Lamme VA, Neumann H, Roelfsema PR The role of attention in figureground segregation in areas V1 and V4 of the visual cortex. Neuron 75: 143-156, 2012.

[PubMed: 22794268]

Prins N, Kingdom F Palamedes: Matlab routines for analyzing psychophysical data. http://www.palamedestoolbox.org, 2009.

Purushothaman G, Marion R, Li K, Casagrande VA Gating and control of primary visual cortex by pulvinar. Nat Neurosci 15: 905-912, 2012. [PMCID: PMC3430824] [PubMed: 22561455]

Roelfsema PR, Lamme VA, Spekreijse H Object-based attention in the primary visual cortex of the macaque monkey. Nature 395: 376-381, 1998. [PubMed: 9759726]

Rossi AF, Pessoa L, Desimone R, Ungerleider LG The prefrontal cortex and the executive control of attention. Exp Brain Res 192: 489-497, 2009. [PMCID: PMC2752881] [PubMed: 19030851] 
Schiller PH, Slocum WM, Kwak MC, Kendall GL, Tehovnik EJ New methods devised specify the size and color of the spots monkeys see when striate cortex (area V1) is electrically stimulated. Proc Natl Acad Sci USA 108: 17809-17814, 2011. [PMCID: PMC3203799] [PubMed: 21987821]

Schiller PH, Tehovnik EJ Visual prosthesis. Perception 37: 1529-1559, 2008. [PMCID: PMC2801810] [PubMed: 19065857]

Silvanto J, Cowey A, Lavie N, Walsh V Striate cortex (V1) activity gates awareness of motion. Nat Neurosci 8: 143-144, 2005. [PubMed: 15643428]

Super H, Roelfsema PR Chronic multiunit recordings in behaving animals: advantages and limitations. Prog Brain Res 147: 263-282, 2005. [PubMed: 15581712]

Super H, Spekreijse H, Lamme VA Two distinct modes of sensory processing observed in monkey primary visual cortex (V1). Nat Neurosci 4: 304-310, 2001. [PubMed: 11224548]

Tehovnik EJ. Electrical stimulation of neural tissue to evoke behavioral responses. J Neurosci Methods 65 : 1-17, 1996. [PubMed: 8815302]

Tehovnik EJ, Slocum WM, Carvey CE, Schiller PH Phosphene induction and the generation of saccadic eye movements by striate cortex. J Neurophysiol 93: 1-19, 2005. [PubMed: 15371496]

Tehovnik EJ, Slocum WM, Schiller PH Saccadic eye movements evoked by microstimulation of striate cortex. Eur J Neurosci 17: 870-878, 2003. [PubMed: 12603277]

Vangkilde S, Coull JT, Bundesen C Great expectations: temporal expectation modulates perceptual processing speed. J Exp Psychol Hum Percept Perform 38: 1183-1191, 2012. [PubMed: 22250866]

Walsh V, Cowey A Magnetic stimulation studies of visual cognition. Trends Cogn Sci 2: 103-110, 1998. [PubMed: 21227086]

Walsh V, Cowey A Transcranial magnetic stimulation and cognitive neuroscience. Nat Rev Neurosci 1: 73 -80, 2000. [PubMed: 11252771]

Watson AB, Pelli DG QUEST: a Bayesian adaptive psychometric method. Atten Percept Psychophys 33: 113-120, 1983.

Zeki S. The disunity of consciousness. Trends Cogn Sci 7: 214-218, 2003. [PubMed: 12757823]

Figures and Tables 
Fig. 1.

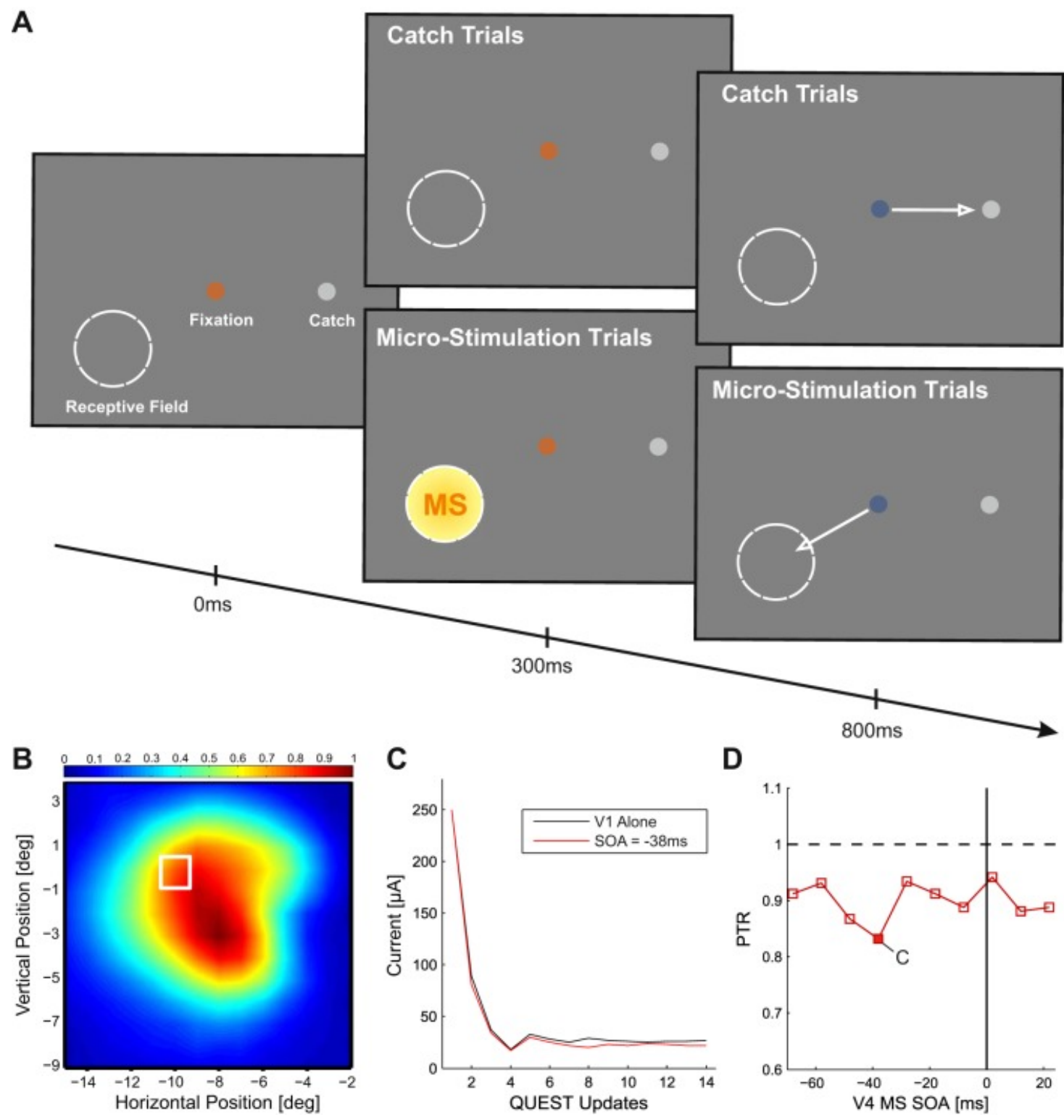

Phosphene detection task. A: after an initial period of $300 \mathrm{~ms}$ of fixation, a train of pulses was delivered to $\mathrm{V} 1$ to evoke a phosphene at the retinotopic location of the receptive field (RF) of the stimulated cells (circle). We also presented a "catch dot" that was the target of the saccade on trials without microstimulation (MS). After a delay of $500 \mathrm{~ms}$, the fixation point changed color, cuing the monkeys to make a saccade. Saccades to the RF in MS trials and to the catch dot on trials without MS were followed by a juice reward. $B$ : overlap between RFs of stimulated neurons in V1 and V4 in an example session with monkey $C$. White square indicates the RF of the neurons in V1; the heatmap indicates the RF of the neurons in V4. $C$ : example of QUEST staircase that was used to determine threshold of phosphenes elicited in V1 in the V1-only (black) condition and in the V1/V4-MS condition with a stimulus-onset asynchrony (SOA) of -38 ms (red). D: relationship between the phosphene threshold ratio (PTR) in V1 and the SOA between subthreshold MS in V4 and MS in V1. Negative values on $x$-axis indicate that V4-MS preceded V1-MS. $y$-Axis shows PTR, the ratio between V1-MS detection thresholds with and without V4-MS. 
Fig. 2.

A

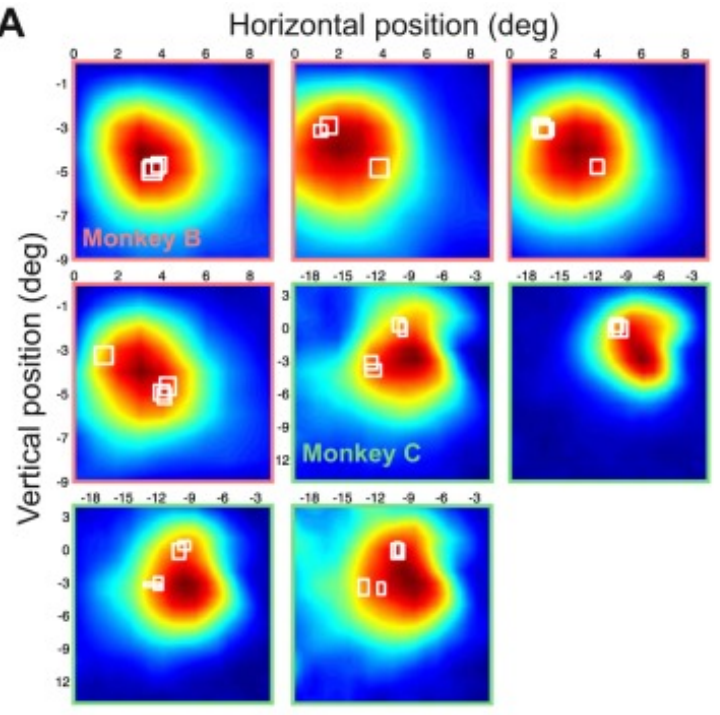

D

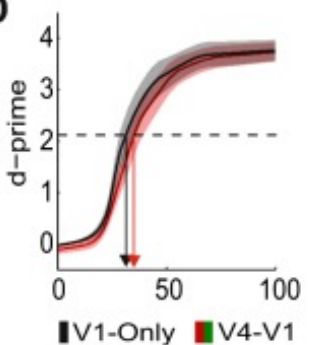

Monkey B

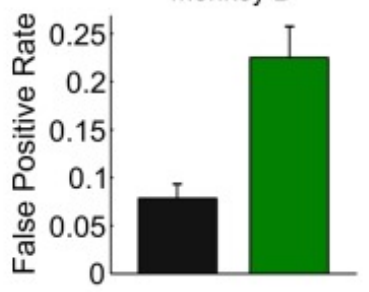

FPR V4 FPR

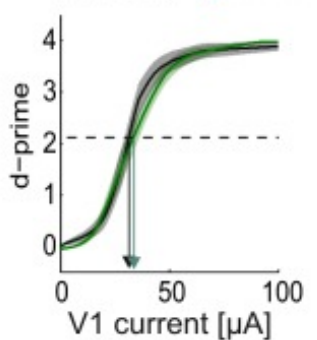

B

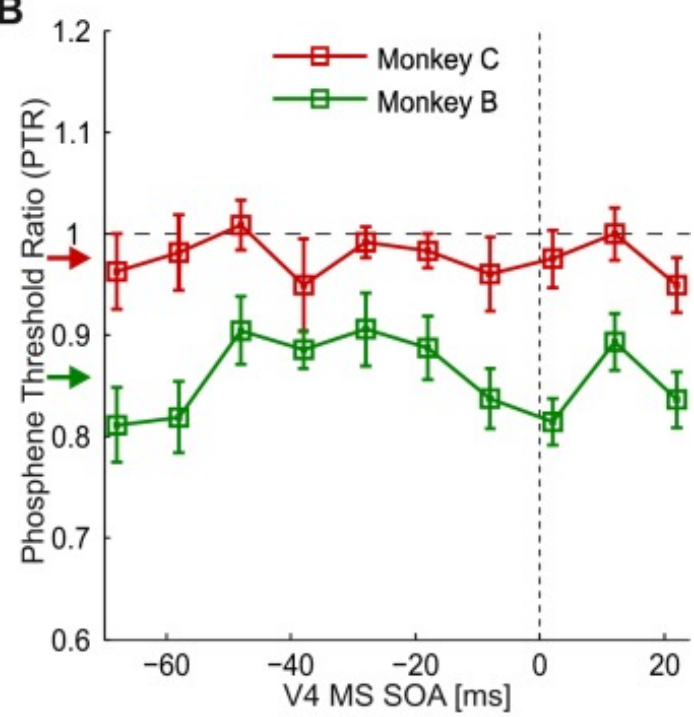

E

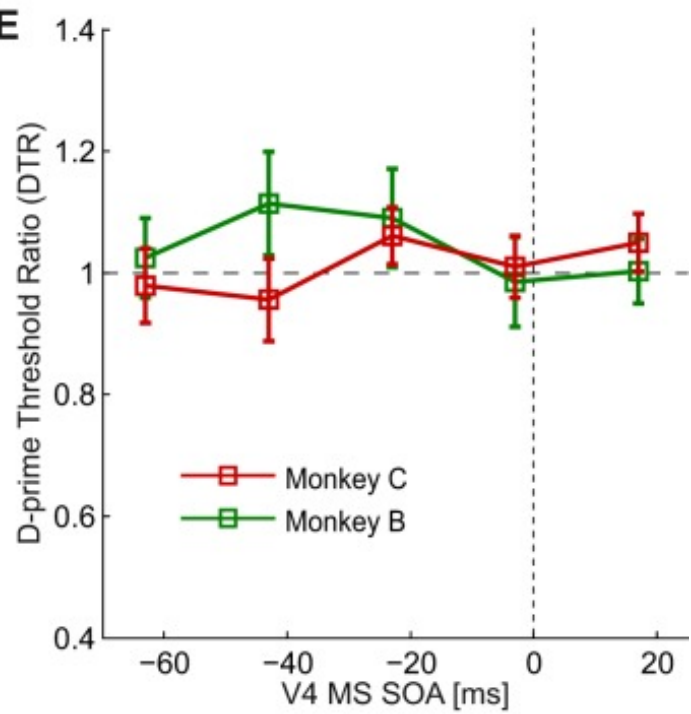

Influence of V4-MS on the detection of phosphenes evoked in V1. A: all combinations of V1- and V4-RFs for monkeys $B$ and $C$ that were tested in the double MS experiment. Color maps show V4-RFs, and white squares illustrate V1-RFs. Color scale as in Fig. 1B. . B: average dependence of PTR in V1 on SOA across all sessions. Negative values on $x$-axis indicate that V4-MS preceded V1-MS. PTR is defined as the threshold with V4-MS expressed as a fraction of the threshold in V1 without V4-MS. Error bars indicate SE across sessions. Colored arrows on $y$-axis indicate $\mathrm{PTR}_{\mathrm{Mean}}$. $C$ : false positive rate (FPR) is the fraction of the trials when monkeys made a saccade toward the RF in catch trials without (black bars) or with V4-MS (colored bars). Red, monkey $C$; green, monkey $B$. Error bars indicate SE across sessions. $D$ : average $d$-prime as function of V1-MS current in the V1-only (black line) and the V4/V1 combined MS condition (colored line). Red, monkey $C$; green, monkey $B$. Shaded areas indicate SE across sessions. E: average dependence of V1 $d$-prime threshold ratio (DTR) on SOA across all sessions. Negative values on $x$-axis indicate that V4-MS preceded V1-MS. DTR is defined as the current in V1 that yields a $d$-prime value of 1 in trials with V4-MS expressed as the fraction of V1 current that yields a $d$-prime of 1 without V4-MS. Error bars indicate SE across sessions. In this analysis adjacent pairs of SOAs were combined in bins to have sufficient data for the computation. 
Fig. 3.

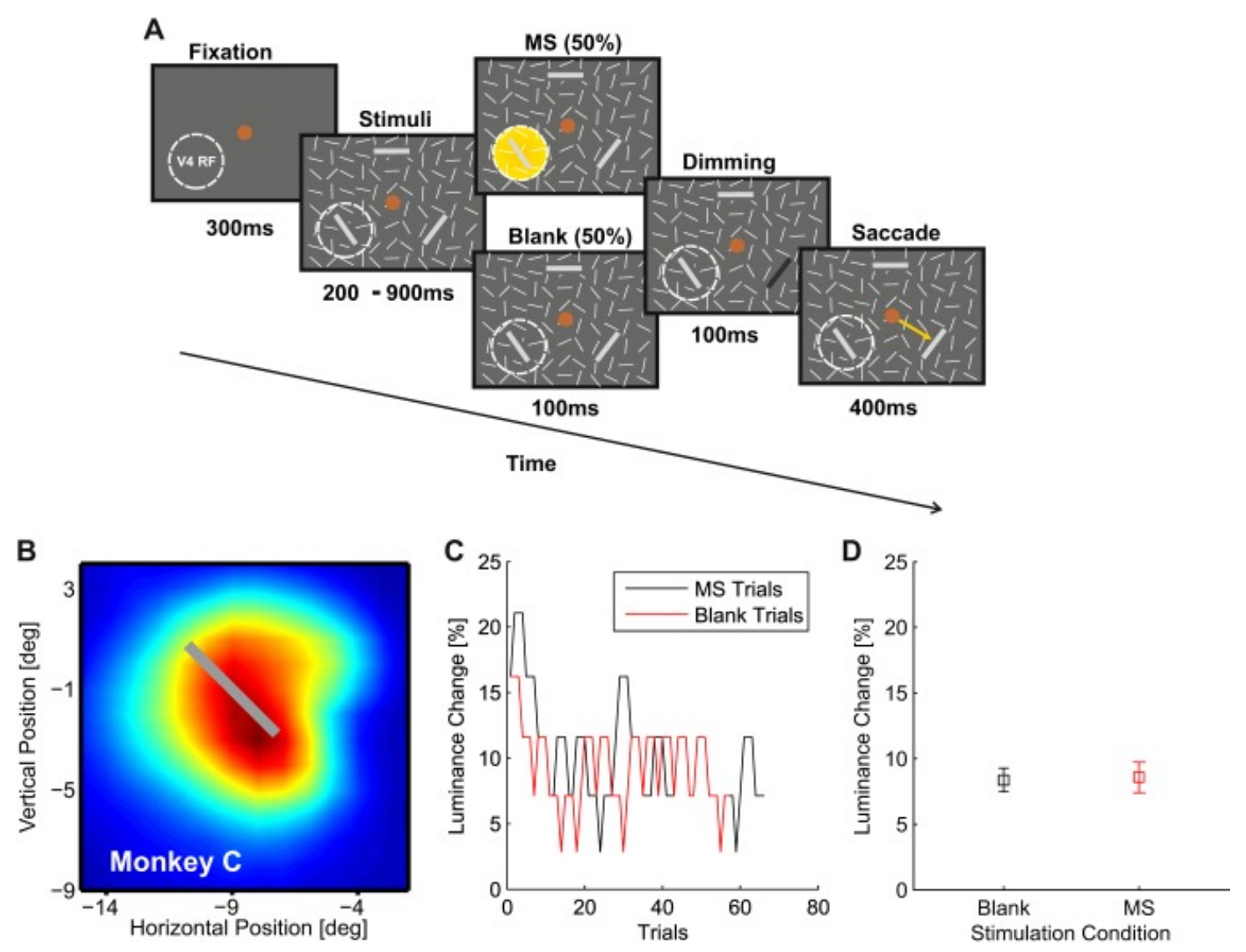

Dimming detection task. $A$ : after $300 \mathrm{~ms}$ of fixation, 3 larger bars were presented among smaller distractor bars. One of the larger bars overlapped with a V4-RF. In half of the trials a train of 20 pulses (total duration $100 \mathrm{~ms}$ ) with a current corresponding to $50 \%$ of the phosphene detection threshold was delivered to V4. After the end of the pulse train, 1 of the bars dimmed for $100 \mathrm{~ms}$ and monkeys had to make a saccade toward this bar. The luminance decrement was controlled by a staircase that kept performance at $79.4 \%$. B: overlap between the RF of the stimulated V4 recording site and the bar in an example session. Color scale as in Fig. $1 B$. $C$ : staircases of an example session, both for the blank (i.e., no MS) and the MS condition with a bar in the stimulated V4-RF. D: thresholds for blank and MS conditions of the example session computed as the mean of the last 15 reversals. Error bars indicate SE of the staircases. 
Fig. 4.
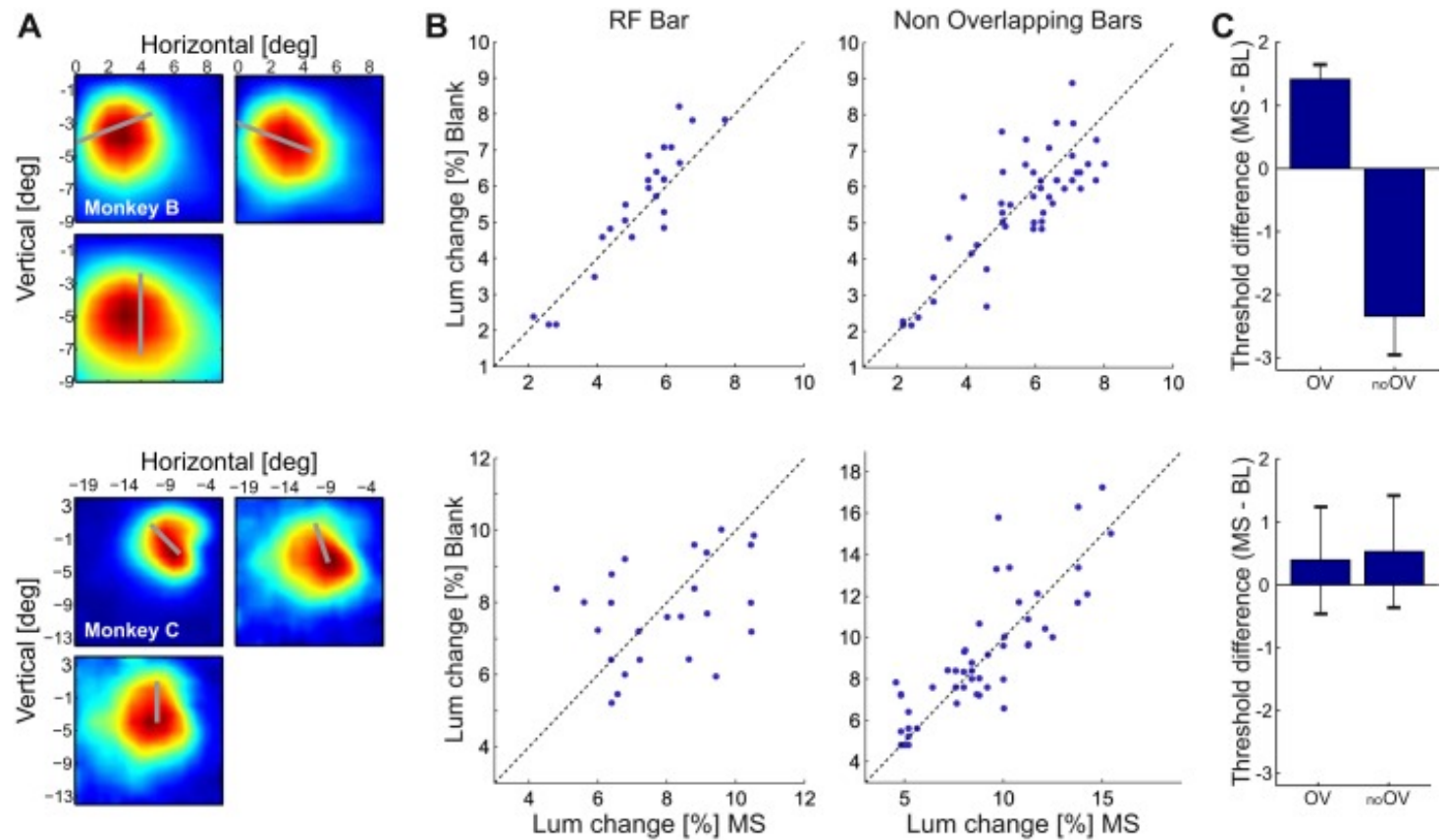

Effect of V4-MS on dimming detection thresholds. $A$ : RFs of V4 recording sites in the dimming detection task relative to 1 of the target bars (gray bars). Color scale as in Fig. 1B. $B$ : dimming detection thresholds for the V4-MS trials ( $x$-axis) and the blank trials ( $y$-axis) for the bar in the RF (left) and the 2 bars outside the RF (right) in monkey B (top) and monkey C (bottom). C: sessions with suprathreshold V4 stimulation (stimulation current was set at $200 \%$ of the threshold). We computed the difference between dimming detection thresholds ( $\%$ contrast change) for bars overlapping with the stimulated V4-RF (OV) and nonoverlapping bars (no OV). Shown are average results of 3 sessions in monkey $B$ and 2 sessions in monkey $C$.

Articles from Journal of Neurophysiology are provided here courtesy of American Physiological Society 